\title{
Late stage definitive endodermal differentiation can be defined by Daf1 expression
}

\author{
Soichiro Ogaki ${ }^{1,2,3}$, Hisayoshi Omori ${ }^{2}$, Mayu Morooka ${ }^{2}$, Nobuaki Shiraki ${ }^{1}$, Seiichi Ishida ${ }^{3}$ and Shoen Kume ${ }^{1,2^{*}}$
}

\begin{abstract}
Background: Definitive endoderm (DE) gives rise to the respiratory apparatus and digestive tract. Sox17 and Cxcr4 are useful markers of the DE. Previously, we identified a novel DE marker, Decay accelerating factor 1(Daf1/CD55), by identifying DE specific genes from the expression profile of DE derived from mouse embryonic stem cells (ESCs) by microarray analysis, and in situ hybridization of early embryos. Daf1 is expressed in a subpopulation of E-cadherin + Cxcr4+ DE cells. The characteristics of the Daf1-expressing cells during DE differentiation has not been examined.
\end{abstract}

Results: In this report, we utilized the ESC differentiation system to examine the characteristics of Daf1-expressing DE cells. We found that Daf1 expression could discriminate late DE from early DE. Early DE cells are Daf1-negative (DE-) and late DE cells are Daf1-positive (DE+). We also found that Daf1+ late DE cells show low proliferative and low cell matrix adhesive characteristics. Furthermore, the purified SOX1 $1{ }^{\text {low }}$ early DE cells gave rise to Daf1+ Sox $17^{\text {high }}$ late DE cells.

Conclusion: Daf1-expressing late definitive endoderm proliferates slowly and show low adhesive capacity.

Keywords: Pluripotent stem cell, Definitive endoderm, Daf1, In vitro differentiation, Proliferation, Adhesion

\section{Background}

The definitive endoderm (DE) gives rise to the gastrointestinal and digestive system. In the mouse embryo, the DE progenitors reside at the posterior region of the epiblast that derived from the inner cell mass [1]. During gastrulation, as the cells ingress through the primitive streak, the epiblast segregates into the three germ layers that form the somatic cell lineages of the ectoderm, mesoderm, and definitive endoderm (DE). DE arises from the Forkhead box A2 (Foxa2)-expressing anterior primitive streak (APS) [2-5] and is then regionalized into the fore-, mid-, and hindgut [6].

The DE is identified by the expression of SRY (sex-determining region Y)-box 17 (Sox17) [7-9] and chemokine (C-X-C motif) receptor 4 (Cxcr4) [10-12]. Sox17 mutant

\footnotetext{
* Correspondence: skume@bio.titech.ac.jp

${ }^{1}$ School of Life Science and Technology, Tokyo Institute of Technology, 4259-B-25 Nagatsuta-cho, Midori-ku, Yokohama, Kanagawa 226-8501, Japan

${ }^{2}$ Stem Cell Biology, Institute of Molecular Embryology and Genetics, Kumamoto University, Honjo 2-2-1, Kumamoto 860-0811, Japan

Full list of author information is available at the end of the article
}

mouse embryos have a reduced DE, apoptosis of the foregut, and abnormal morphogenesis of the mid- and hindgut [9]. Sox17 is also required for the assembly of the basement membrane, as the Sox17 mutant embryo fails to segregate the DE from the mesoderm [13]. Activin is a frequently used inducer for DE differentiation from pluripotent stem cells, embryonic stem cells (ESCs), and induced pluripotent stem cells (iPSCs) [14-16]. When SOX17 is overexpressed, human ESCs spontaneously differentiate into the DE, independent of Activin [17]. In zebrafish embryos, cxcr4a regulates directional migration $[11,18]$ and DE proliferation during gastrulation [19]. In chick embryos, Cxcr4 is expressed in the DE and angioblasts. Cxcr4 and its ligand Cxcl12 form a reciprocal signaling loop that triggers angioblast migration to the pancreatic endoderm and induces pancreatic development. Inhibition of Cxcr4 suppresses angioblast migration into the pancreatic endoderm region. As a result, the size of the pancreas decreases [10]. Although Cxcr4 is also expressed in the mesodermal cells, it is often used in combination with E-cadherin for purifying ESC-derived DE cells using flow cytometry [12]. 
Daf1 is an inhibitor of complementary activation [20]. Daf1 is expressed by immune cells and DE-derived tissue, such as intestine and airway [21]. Using microarray analysis and in situ hybridization, we previously identified Daf1 as a DE cell surface marker based on its expression in ESC-derived and embryonic DE. Daf1 is also expressed in pancreatic progenitor cells $[22,23]$. However, the role of Daf1 in the DE is not well understood. In this study, we found that the DE population that expresses Daf1 (Daf1 + DE) has slow cell cycling and low cell-matrix adhesive characteristics. Furthermore, Daf1-negative DE cells (Daf1-DE) turn out to be Foxa $2+$ Sox $17^{\text {low }}$ cells and Daf1-positive DE $\left(\right.$ Daf1 + DE) cells to be Foxa $2+$ SOX17 $7^{\text {high }}$ cells. Our results therefore suggest that E-cadherin + Cxcr4 + DE is composed of two populations: Sox $17^{\text {low }}$ early DE and Sox $17^{\text {high }}$ late DE. Sox17 high late DE cells were positive for Daf1, and were slow proliferative and low cell-matrix adhesive cells.

\section{Results}

Daf1 + DE are slowly proliferating cells

Previously, we reported Daf1 as a surface marker, expressed in a subpopulation of $\mathrm{DE}$ [23]. $\mathrm{DE}$ are defined as Ecadherin+/Cxcr4+ cells [12]. When cultured in Activincontaining medium $[24,25]$, ESCs sequentially give rise to APS cells on day4 (defined as E-cadherin+/Pdgfra + cells), then to DE cells (defined as E-cadherin+/Cxcr4+ cells) on day 5 (Fig. 1). A plot of our previous microarray analysis results of the APS and DE cells [23, 24] shows the time dependent expression of Foxa2, Sox17 and Daf1 (Fig. 1a). Foxa2 was highly expressed in the APS and DE. Sox 17 was lowly expressed in the APS and highly expressed in the DE. Daf1 expression was absent in the APS and present in the DE (Fig. 1a). We then analyzed Daf1+ cells for the expression of Nanog, a pluripotent marker, using a mouse Nanog-iPS cell line bearing a green fluorescent protein (GFP) reporter driven under Nanog promoter. Daf1-positive cells turned out to be Nanog/GFP negative (Additional file 1). Taken together, Daf1 marks a subpopulation of DE and is first expressed in the DE, but not earlier in the APS.

To identify the differences between Daf1 + DE and Daf1-DE cells, we purified Daf1+/-DE cells and compared their properties. Real time PCR analysis of the sorted Daf1+/-DE cells confirmed Daf1 expression is enriched in Daf1 + DE (Fig. 1b, c). Expressions of an $M$ phase marker, phosphorylated histone $\mathrm{H} 3$ (pH3) or Proliferating Cell Nuclear Antigen (PCNA) that marks proliferating cells at every phase of the cell except G0 were enriched in Daf1-DE than in Daf1 + DE cells, revealed by western blot analysis (Fig. 1d). We analyzed the cell cycle phases and found that Daf1 + DE cells showed a longer Go/G1 phase and shorter $\mathrm{S}, \mathrm{M} / \mathrm{G} 2$ phase compared to Daf1-DE cells (Fig. 1e, Additional file 2). This suggested that cell proliferation was decreased in Daf1 + DE.

\section{Daf1 + DE are low adhesive cells}

Next, we performed flow cytometry using antibody against Dafland plated at a same cell number of the sorted DE cells (Daf1-DE or Daf1 + DE) onto matrigel-pre-coated dishes (Fig. 2a). After 24 h culture, the Daf1+ DE gave rise to fewer cell number compared to that of Daf1-DE (Fig. 2b, c). The results suggested a higher level of cell death and/ or lower plating efficiency of Daf1 + DE cells. We then examined cell-matrix adhesion and the extent of apoptosis, in which cells were allowed to adhere to the plate for 90 mins [26, 27] [28]. Our results revealed that Daf1 + DE cells showed a lower matrix adhesion ratio than Daf1-DE (Fig. 2d), and that Daf1 + DE cells were more apoptotic and showed a higher Cleaved Caspase3/7+ ratio (Fig. 2e) than Daf1-DE. Cells bind to extracellular matrix (ECM) through the ECM receptor, integrin [29, 30]. We found that expression of several subtypes of the Integrin genes, such as $\operatorname{Itg} \alpha 1$, Itg $\alpha 3$, and $\operatorname{Itg} \alpha 8$, was down-regulated in Daf1 + DE cells (Fig. 2f). Our results suggest that Daf1 + DE cells have lower adhesion capacity than Daf1-DE cells, which led to a lower plating efficiency and triggered cell death in the attached cells, compared to Daf1-DE cells.

\section{Daf1 is a marker of late stage DE}

To characterize the adherent cells onto Matrigel- precoated dish, we assayed for the expression of the DE markers, Foxa 2 and Sox17, immediately after the adhesion assay (Fig. 3). Interestingly, whereas both Daf1+/-DE populations showed similar capacity for giving rise to Foxa2expressing cells, Daf1 + DE cells showed a higher capacity for giving rise to Sox17-expressing cells (Fig. 3a, b). Foxa2 is expressed in both the APS and DE, whereas Sox17 is expressed at a higher level in the DE. We then confirmed Sox17 protein expression of the sorted Daf1+/-DE cells directly (without plating) by immunocytochemical analysis (Fig. 3c). Sox17 expression was found in a higher proportion and seemingly higher level in the sorted Daf1 + DE cells compared to Daf1-DE cells (Fig. 3c). Western blot analysis confirmed a higher level of Sox17 expression in the Daf1 + DE cells (Fig. 3d). Since Foxa2 expression is detected prior to that of Sox17, we then asked if Daf1-DE cells represent early DE and Daf1 + DE cells represent late DE, by examining if Daf1-DE cells differentiate into Daf1 + DE. Daf1+/-DE cells sorted by flow cytometry (purity $>97.8 \%$, Daf1 and Cxcr4 expression profiles shown in Fig. 3e) were re-cultured on mouse embryonic fibroblast (MEF) feeders with Activin containing endoderm differentiation medium. We examined the expression of Daf1. To exclude MEF feeders, we analyzed E-cadherin + cells. Almost all of the Daf1-DE cells acquired Daf1 expression over the course of $24 \mathrm{~h}$ and gave rise to Daf1 + DE (>96.7\%), whereas Daf1 + DE cells did not turn into Daf1-DE (Fig. 3e). Taken together, these results suggest that Daf1-DE represent early and Daf1 + DE 


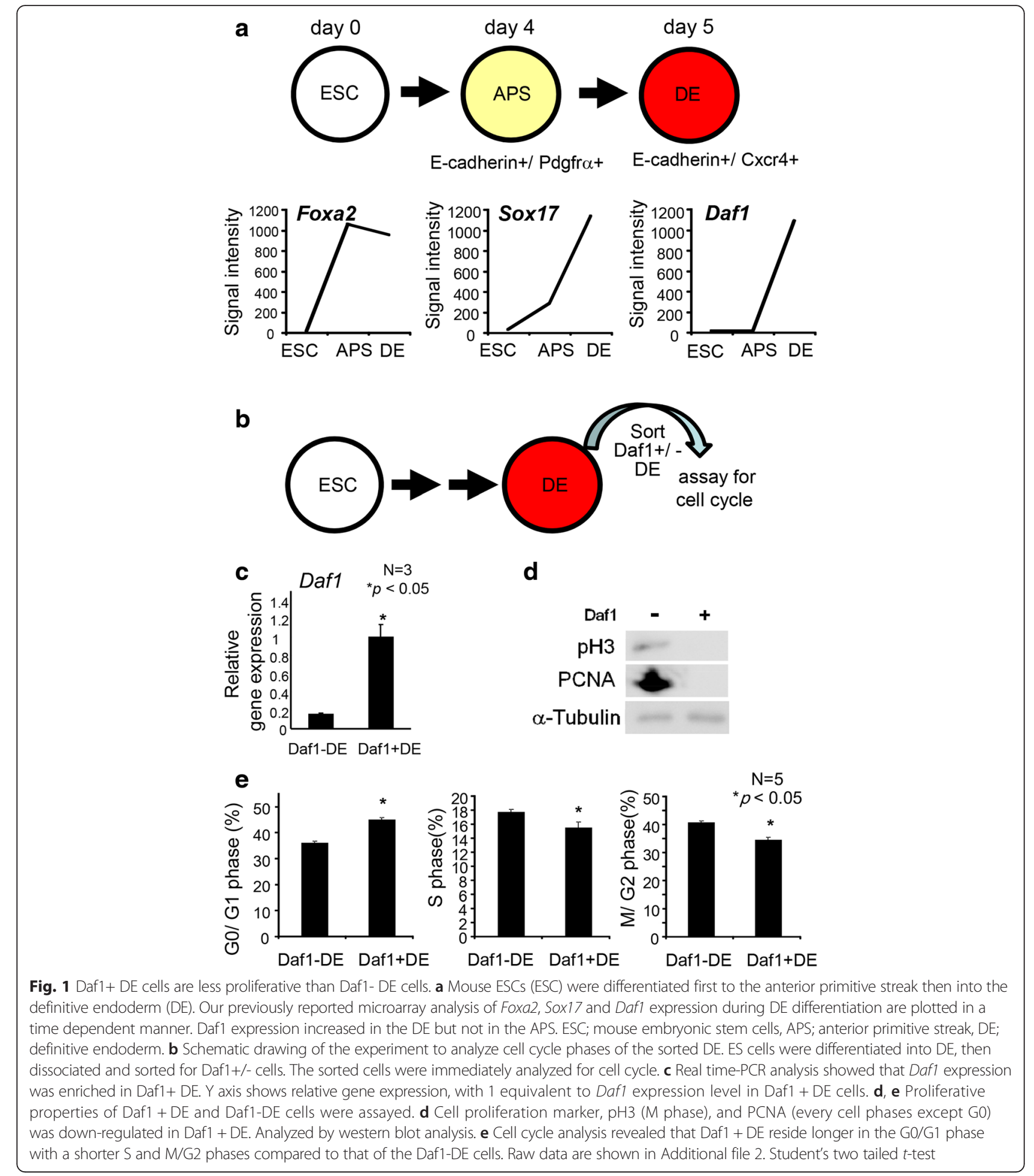

represent late DE cells during DE differentiation and that Daf1-DE become Daf1 + DE cells (Fig. 3f).

Daf1+/-DE cells can give rise to pancreatic and intestinal fates We identified that Daf1-DE cells acquired Daf1 expression and turned into Daf1 + DE. If Daf1-DE represents early DE and Daf1 + DE represents late DE, both DE cells could give rise to regional endoderm derivatives of the gut. We next examined the abilities of the Daf1+/-DE cells to differentiate into pancreatic or intestinal cells. To visualize pancreatic differentiation, we used $P d x 1 /$ GFP mouse ESCs, in which GFP expression is driven by the 

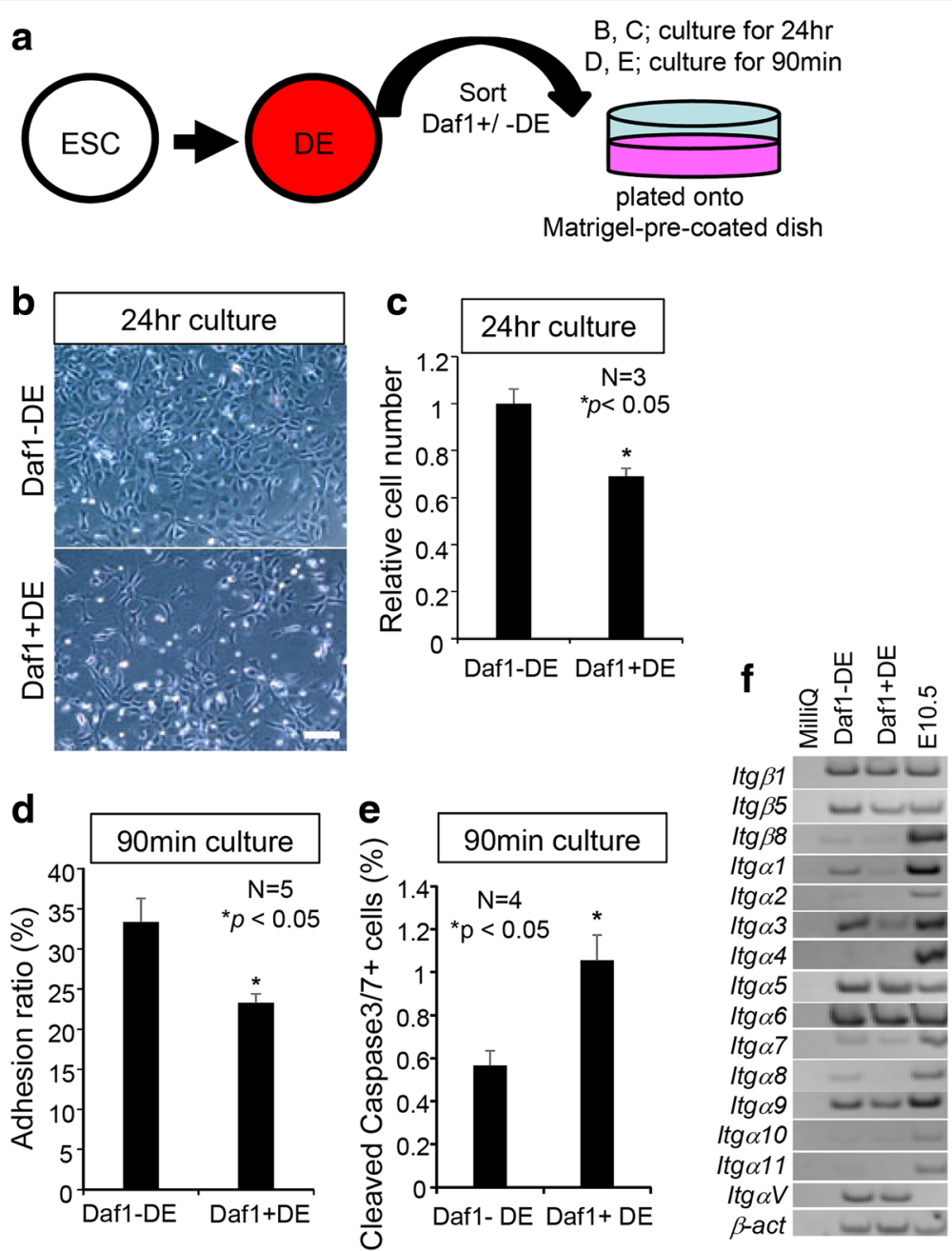

Fig. 2 Daf1+ DE cells have lower cell-matrix adhesion than Daf1- DE cells. a A schematic drawing showing experimental design. The sorted DE cells were plated onto matrigel-pre-coated dishes and cultured for $24 \mathrm{~h}(\mathbf{b}, \mathbf{c})$ or $90 \mathrm{~min}(\mathbf{d}, \mathrm{e})$. $\mathbf{b}$ Transmission images of the cells derived from the Daf1+/-DE cells after cultured for $24 \mathrm{~h}$. c After $24 \mathrm{~h}$ culture, the Daf1 + DE cells gave rise to fewer cells. $\mathbf{d}$ Cell-matrix adhesion assay, after 90 min culture. Quantification of the attached cells. The adhesion ability of Daf1 + DE cells onto matrigel is weaker than that of the Daf1-DE cells. e Quantification of Cleaved caspase3/7+ apoptotic cells. The Daf1 + DE cells gave rise to a larger proportion of apoptotic cells. $\mathbf{f}$ The Integrin expression profiles of the DE cells detected by RT-PCR analysis. Student's two tailed t-test. Scale bars; $100 \mu \mathrm{m}$

$P d x 1$ promoter [25]. We isolated Daf1+/-DE cells by flow cytometry (purity $>98 \%$ ), seeded the cells on MEF feeders, and cultured in the presence of Activin and FGF2 (Fig. 4a) [25]. We found that both Daf1- DE and Daf1+ cells gave rise to $P d x 1 / \mathrm{GFP}+$ cells, after plated for 3 days (day 8). The Daf1 + DE-derived cells yielded less $P d x 1 /$ GFP cells compared to that of the Daf1-DE cells (Fig. 4b, c). This discrepancy was probably due to a lower proliferation of the Daf1 + DE cells compared to Daf1-DE cells. Since the pancreas derives from the anterior DE, we then asked if the Daf1+/-DE cells could also differentiate into the posterior DE derivative of the intestine. DE derived from R1 ESCs were sorted and seeded onto MEF feeders and cultured at the presence of BIO and DAPT $[16,31]$. After five days, both Daf1-DE and Daf1 + DE cells differentiated into $\mathrm{Cdx} 2+$ intestinal cells. Similar to pancreatic differentiation, Daf1 + DE cells are less proliferative and gave rise to small $\mathrm{Cdx} 2+$ colonies than Daf1-DE cells (Fig. 4c). Our results therefore suggest that both Daf1+/- DEs are capable of differentiating into the endodermal derivatives of the pancreas and intestine.

\section{Discussion}

Previously, we identified that Daf1 is expressed in the DE. Here, we identified Daf1 as a late DE marker. Daf1 is an inhibitor of complement activation [20]. Daf1 is expressed in the kidney, spleen, testis, intestine, and bronchi of the adult mouse [21]. Daf1 deficiency is reported in autoimmune hemolytic anemia patients [32]. In Daf1 knockout mice, IFN- $\gamma$ expression increases, 

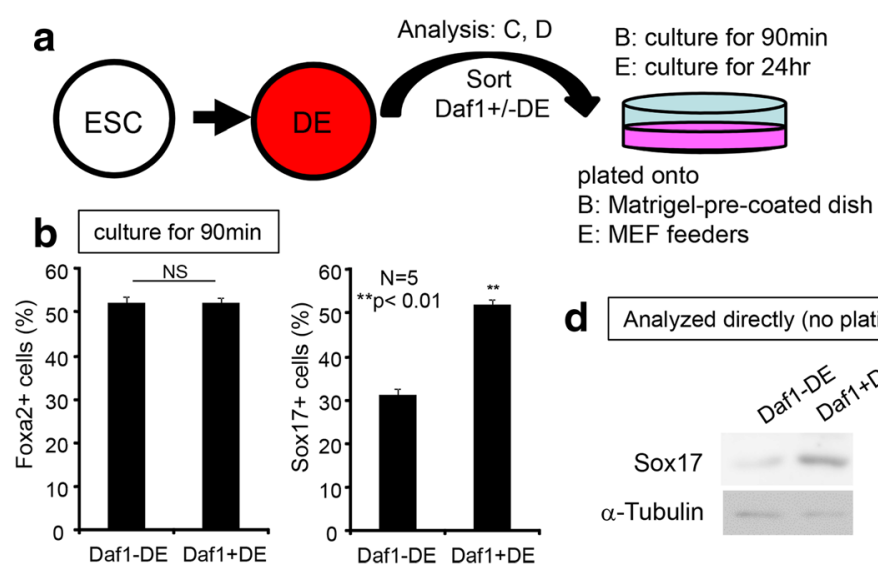

d Analyzed directly (no plating)
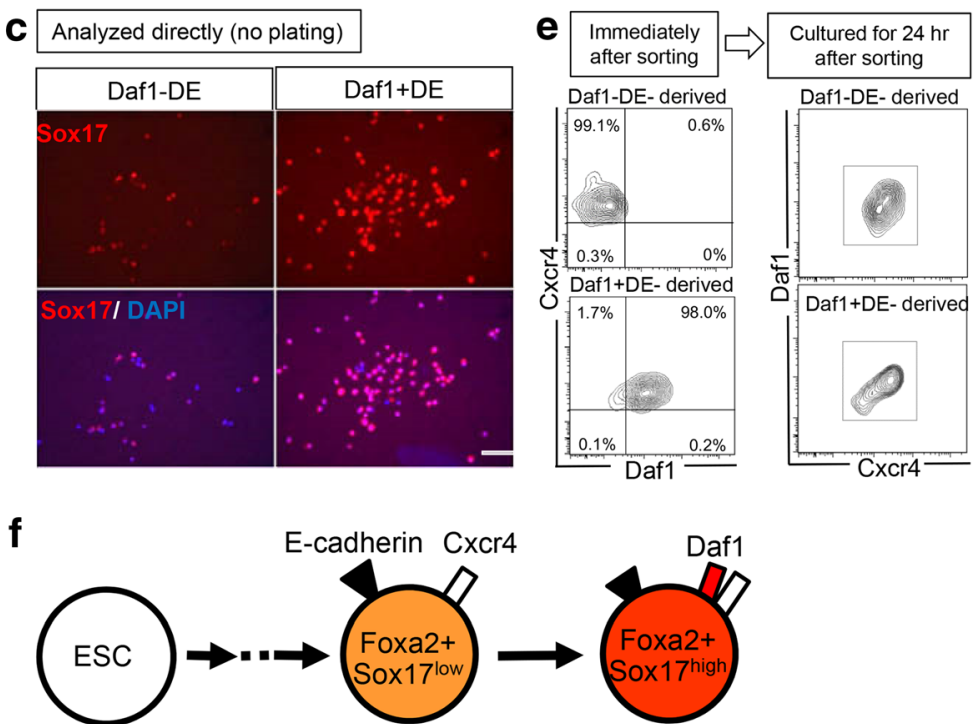

Fig. 3 Daf1-DE is the progenitor of Daf1+DE. a A schematic drawing showing experimental design. $\mathbf{b}$ Quantifications of Foxa2+ and Sox17+ cells by immunocytochemistry after 90 min cell-matrix adhesion assay. $\mathbf{c}, \mathbf{d}$ The proportion of cells expressing Sox17 was higher in the Daf1 + DE as detected by immunocytochemical analysis (c), and the level of Sox17 expression was higher in the Daf1 + DE as detected by western blot (d), of the sorted DE cells (without plating). e Flow cytometry analysis of the descendent cells of Daf1-DE and Daf1 + DE. Left panels: Daf1, Cxcr4 expression profiles of the sorted Daf1+/- DE cells. Right panels: both Daf1-DE and Daf1 + DE acquired Daf1 expression after 24 h. $\mathbf{f}$ The scheme of Fig. 3. Sox17 ${ }^{\text {low }}$ Daf1-DE differentiated into Sox1 $17^{\text {high }}$ Daf1 + DE. Student's two tailed t-test. Scale bar; $100 \mu \mathrm{m}$

resulting in enhanced $\mathrm{T}$ cell response autoimmunity [33]. However, gastrointestinal-tract develops normally in Daf1 knockout mice. Here, we examined the detailed expression patterns during DE differentiation using ESCs.

DE cells are defined as E-cadherin+/Cxcr4+ cells [12]. However, both E-cadherin and Cxcr4 are also expressed in the primitive streak $[34,35]$. Therefore, the use of E-cadherin+/Cxcr4+ as a marker to define the DE cells is confined to a limited time window. Moreover, Ecadherin+/Cxcr4+ DE cells are a heterogeneous population. Here we used Daf1 to characterize a subpopulation of E-cadherin+/Cxcr4+ DE cells. We revealed that both Daf1-DE cells represent early DE and Daf1 + DE represent late DE. Daf1-DE and Daf1 + DE cells can give rise to the pancreatic and intestinal lineages. Daf1 + DE formed small colonies, due to their less proliferative and low adhesive characteristics than that of Daf1-DE cells (Fig. 5). A slight decrease in S, M/G2 phase and increase in G0/G1 phase in Daf1 + DE cells might reflect their property as more differentiated cells. Daf1 + DE cells seem to differentiate as efficiently into Pdx1-expressing cells, but not as efficiently into Cdx2-expressing cells, compared to Daf1-DE cells. This might due to a partial loss in differentiation potency of Daf1+ DE cells into the intestinal fate. We previously reported that regional-specific endodermal fates are determined sequentially in the order of stomach, intestine and pancreas, in the chick embryos [36]. It is possible that Daf1 + DE gradually lose potency to differentiate into intestinal lineages, but retains differentiation potency into pancreatic lineages, compared to that of Daf1-DE. 

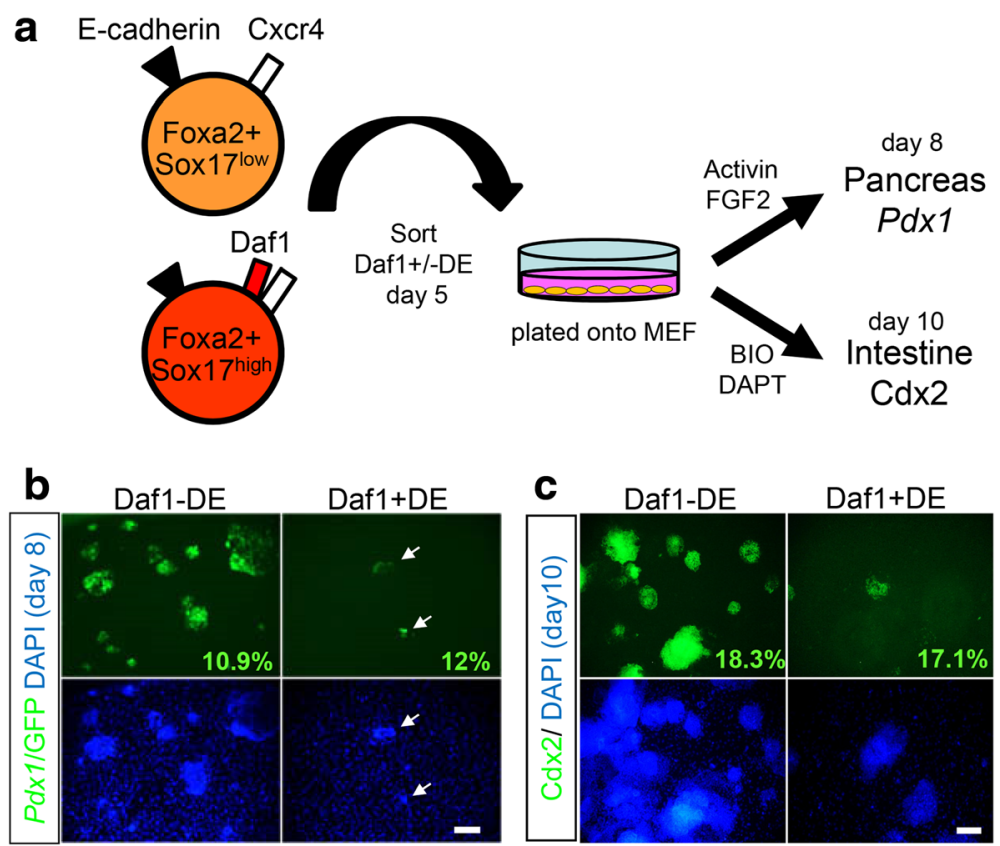

Fig. 4 Both Daf1+/- could differentiate into pancreatic and intestinal fates. a A schematic drawing showing experimental design. DE was sorted on day 5. The sorted cells were plated onto MEF feeders and cultured with pancreatic and intestinal differentiation medium. Daf1- or Daf1 + DE cells differentiated into Pdx1/GFP+ cells after 4 days culture (b) and Cdx2+ cells after 5 days culture (c). $\mathbf{b}$ Arrows depict small colonies of Daf1 + DE-derived Pdx1/GFP+ cells. Numbers depict differentiation ratios: Daf1-DE-derived Pdx1/GFP+ cells; $10.9 \%$, Daf1 + DE-derived Pdx1/ GFP+ cells; $12 \%$ (day 9). c Numbers depict differentiation ratios: Daf1-DE-derived Cdx2+ cells; $18.3 \%$, Daf1 + DE-derived Cdx2+ cells; $17.1 \%$ (day 10). Scale bar; $100 \mu \mathrm{m}$

Cell-matrix adhesion is also necessary for cell differentiation [37, 38]. Integrin expression promotes DE differentiation from human pluripotent stem cells [39]. Integrin is a receptor of extracellular matrix expressed in the cells, which enables binding of the cells to the extracellular matrix. Itg $\alpha 5$ and Itg $\alpha \mathrm{V}$ are $\mathrm{DE}$-specific Integrins. Knockdown of either Itg $\alpha 5$ or Itg $\alpha \mathrm{V}$ inhibits DE differentiation [39]. Both Daf1- and Daf1 + DE cells express $\operatorname{Itg} \alpha 5$ and $\operatorname{Itg} \alpha \mathrm{V}$. We found that the expressions of Itg $\alpha 1$, Itg $\alpha 3$, and Itg $\alpha 8$ decreased in Daf1 + DE cells. Itg $\alpha 1$ is an attachment molecule of the DE [40]. Itg $\alpha 3$ is expressed in the DE and expression decreases in the
Foxa2 null mouse embryo [41]. Itg $\alpha 8$ null mice have abnormal lung morphogenesis [42]. These Daf1-DE specific integrins could regulate DE differentiation and modulate their behavior. The integrins are known to show distinct ligand binding specificities among the superfamily members. $\operatorname{Itg} \alpha 1 \beta 1$, $\operatorname{tg} \alpha 3 \beta 1$ bind specifically to laminin and $\operatorname{Itg} \alpha 8 \beta 1$ binds specifically to fibronectin [43]. The lowered expression of $\operatorname{Itg} \alpha 1$, Itg $\alpha 3$ might explain the decreased adhesion of Daf1 + DE cells to Matrigel, which composed mainly of laminin. The decrease in adhesion to matrix might reflect the developmental transition from early to late DE.

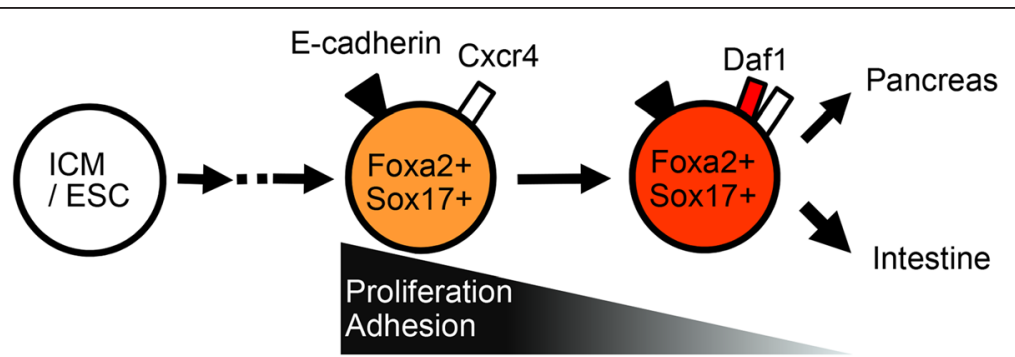

Fig. 5 Proposed role of Daf1 in DE differentiation. ICM/ESC differentiated into the E-cadherin + Cxcr4 + Foxa2 + Sox17 ${ }^{\text {low } D a f 1-~ e a r l y ~ D E . ~ T h e s e ~ c e l l s ~}$ then differentiated into the E-cadherin $+\mathrm{CXCr} 4+$ Foxa2 + SOX1 $17^{\text {high }}$ Daf1 + late DE. The late DE differentiated into regionalized anterior (pancreatic) and posterior (intestinal) endodermal lineages. Transition from the Daf1-DE to Daf1 + DE is accompanied by restricted cell proliferation and cell-matrix adhesion. ICM; inner cell mass 
We found that Daf1-DE could turn into Daf1 + DE cells. Sox17 expression was higher in Daf1 + DE than in Daf1-DE cells. Furthermore, expression of a primitive streak marker, Brachyury [44], was higher in Daf1-DE cells (SO unpublished). Therefore, E-cadherin+/Cxcr4+ $\mathrm{DE}$ could be a mixed population of both primitive streak and DE cells.

\section{Conclusions}

Our data indicate that DE can be divided into two stages: early and late DEs. Early DE consists of E-cadherin + Cxcr4 + Daf1-Foxa2 + Sox17 ${ }^{\text {low }}$ cells that show higher proliferative activity and higher cell-matrix adhesive capacity. Later on, these DE cells differentiate into E-cadherin + Cxcr4 + Daf1 + Foxa2 + Sox17 ${ }^{\text {high }}$ late DE cells that show a decreased proliferation and low cell-matrix adhesion capacity.

Our findings would contribute to the understandings of the differentiation of the primitive streak and DE during gastrulation.

\section{Methods}

\section{Cell lines}

ESC cell lines (R1, SK7 Pdx1/GFP) [25] or a mouse Nanog iPS cell line (20D-17) [45], were maintained on a feeder layer of mouse embryonic fibroblasts (MEFs) in Dulbecco's Modified Eagle Medium (DMEM) (Invitrogen) supplemented with human recombinant LIF (1:1000, Wako), $10 \%$ fetal bovine serum (FBS, Hyclone), 2 mM L-glutamine (L-Gln, Nacalai Tesque), $100 \mathrm{mM}$ non-essential amino acids (NEAA, Invitrogen), $50 \mathrm{U} / \mathrm{mL}$ penicillin, $50 \mathrm{mg} / \mathrm{ml}$ streptomycin (PS, Nacalai Tesque), and $100 \mu \mathrm{M} 2$ mercaptoethanol (2-ME, Sigma-Aldrich) in $5 \% \mathrm{CO}_{2}$.

\section{Differentiation of ESCs}

DE differentiation: ESCs or iPSCs $\left(10^{4}\right.$ cells $\left./ \mathrm{ml}\right)$ were seeded onto mitomycin C (Sigma)-treated M15 feeders, and cultured in the presence of $10 \mathrm{ng} / \mathrm{ml}$ Activin (R\&D systems) in DMEM containing 10 \% FBS, 2 mM L-Gln, $100 \mathrm{mM}$ NEAA, PS, and $100 \mu \mathrm{M}$ 2-ME. Pancreatic differentiation: Sorted DE cells were seeded onto MEF feeders and cultured with DE differentiation medium supplemented with $5 \mathrm{ng} / \mathrm{ml} \mathrm{FGF2} \mathrm{(Peprotech).} \mathrm{Intestinal}$ differentiation: Sorted DE cells were seeded onto MEF feeders and cultured with DMEM (2000 $\mathrm{mg} / \mathrm{ml}$ glucose) and $5 \mu \mathrm{M}$ bromoindirubin-3'-oxime (BIO) (Wako), $10 \mu \mathrm{M} \mathrm{N}$-[(3, 5-diflurophenyl) acetyl]- L-alanyl-2-phenylglycine-1, 1-dimethylethyl ester (DAPT) (Peptide), $10 \%$ Knockout Serum Replacement (KSR)(In vitrogen), 2 mM L-Gln, 100 mM NEAA, PS, and $100 \mu \mathrm{M}$ 2-ME.

\section{Microarray analysis}

Our previously described results were used in the present study [22, 23].

\section{Antibodies}

For immunocytochemical analysis, goat anti-Sox17 antibody (1:100, R\&D systems), rabbit anti-Hnf3b/Foxa2 (1:200, Millipore), mouse anti-Cdx2 (1:500, BioGenex) and rabbit anti-GFP (1:1000, MBL) were used. For flow cytometric analysis, rat anti-E-cadherin (1:500, TaKaRa), biotin anti-Cxcr4 (1:500, BD Biosciences), PE anti-CD55/ Daf1 (1:100, BD Biosciences), PE/Cy7 Streptavidin (1:500, Biolegend) antibodies were used. E-cadherin antibody was labeled by Allophycocyanin Labelling Kit-SH2 (DOJINDO). For Western blot analysis, mouse anti- $\alpha$-tubulin (1:2000, 12G10, Developmental Studies Hybridoma Bank), mouse anti-phospho-Histone H3 (Ser10) antibody (1:500, Millipore), rabbit anti-Sox17 antibody (1:100, Sigma-Aldrich) and mouse anti-PCNA (1:500, Oncogene, NA03-200UG) were used.

\section{Immunocytochemical analysis}

Cells were fixed with $4 \%$ paraformaldehyde (PFA) (Nacalai Tesque) for $5 \mathrm{~min}$. After fixation, cells were permeabilized with $0.1 \%$ TritonX (Nacalai Tesque) for $10 \mathrm{~min}$. Then, cells were blocked with Blocking One (Nacalai Tesque) and stained with antibodies.

\section{Flow cytometry analysis}

Cells were dissociated with Cell Dissociation Buffer (Invitrogen) and stained with the appropriate antibodies. The stained cells were recovered using FACS Aria II (BD Biosciences). Data were recorded using the BD FACS Diva Software program (BD Biosciences) and analyzed using the FlowJo program (Tree Star).

\section{Western blot analysis}

Cells were homogenized in SDS sample buffer $(62.5 \mathrm{mM}$ Tris- $\mathrm{HCl}, 10 \%$ glycerol, 2 \% SDS, pH 6.8). After centrifugation, the supernatants were collected and used as total protein extracts. Total proteins were subjected to $8 \%$ SDS-PAGE and transferred to polyvinylidene difluoride membranes (Immobilon-P Transfer Membrane, Millipore). The membranes were incubated with antibodies listed above. Horseradish peroxidase (HRP) conjugated antirabbit IgG (1:20000, CST) was used as the secondary antibody. Chemiluminescence signals were detected with ECL Plus Western Blotting Detection Reagents (GE Healthcare, Japan).

\section{RT-PCR analysis}

RNA was extracted from the cells using the RNeasy Micro-Kit (QIAGEN) and then $1 \mu \mathrm{g}$ of RNA was reverse transcribed using ReverTra Ace (TOYOBO), ribonuclease inhibitor, recombinant (TOYOBO), and Oligo dT primers (TOYOBO). Primer sequences are shown in Additional file 3. 


\section{Cell cycle analysis}

Cells were dissociated with Cell Dissociation Buffer (Gibco). Dissociated cells were washed with PBS and treated with Vybrant DyeCycle Violet Stain (Life Technologies) for $30 \mathrm{~min}$ at $37^{\circ} \mathrm{C}$. Cells were analyzed by FACS Canto (BD Biosciences).

\section{Cell- matrix adhesion analysis}

The sorted cells were plated onto matrigel-precoated dishes with serum free medium for $90 \mathrm{~min}$. The attached cells were fixed with $4 \%$ PFA for 5 min, then stained with DAPI (1:2000, Roche). Cell counts were performed as previously described [46].

\section{Apoptosis assay}

For the apoptosis assay, caspase-3/7 activity was measured using CellEvent ${ }^{\text {ta }}$ Caspase-3/7 Green Detection Reagent (Invitrogen Life Technologies Co., Carlsbad, CA, USA) according to the manufacturer's protocol.

\section{Additional files}

Additional file 1: Daf1-positive cells are negative for Nanog expression. Mouse Nanog-iPS cells, in which GFP expression is driven by Nanog promoter [45], are differentiated into DE. Cxcr4+/E-cadherin + cells were sorted and analyzed for Daf1 and Nanog-GFP expression. Daf1-positive cells are negative for Nanog expression. (TIF $312 \mathrm{~kb}$ )

Additional file 2: Flow cytometric analyses of the cell cycle. Histograms of flow cytometric analyses of Daf1-DE and Daf1 + DE $(n=5)$ are shown. Cell cycle was analyzed by measuring DNA quantities using DyeCycle. (TIF 314 kb)

Additional file 3: Primer sequences used for RT-PCR analysis. Primer sequences used for detection of gene expression in Fig. 1, 2. (DOCX 15 kb)

\section{Abbreviations}

2-ME, $\beta$-mercaptoethanol; APS, anterior primitive streak; Cxcr4, chemokine (C-X-C motif) receptor 4; Daf1/CD55, decay accelerating factor 1; Daf1-DE, Daf1-negative DE cells; DAPI, 2-(4-amidinophenyl)-1H -indole-6-carboxamidine; DE, definitive endoderm; DMEM, Dulbecco's modified eagle medium; ESC, embryonic stem cells; FBS, fetal bovine serum; FoxA2, Forkhead box A2; GFP, green fluorescent protein; HRP, horseradish peroxidase; iPSC, induced pluripotent stem cell, Daf1 + DE, Daf1-positive DE cells; KSR, knockout serum replacement; L-GIn, L-glutamine; MEF, mouse embryonic fibroblasts; NEAA, non-essential amino acids; PCNA, proliferating cell nuclear antigen; PFA, paraformaldehyde; PH3, phosphorylated histone H3; PS, penicillin \& streptomycin; RT-PCR, reverse transcription-polymerase chain reaction; Sox17, sex-determining region Y-box 17

\section{Acknowledgements}

We thank Dr. T. Seki (Kumamoto University) for maintenance of FACS Aria II. This work was supported by Grants-in-Aid (to S.K, 26253059 and 26670384) from the Ministry of Education, Culture, Sports, Science and Technology (MEXT) Japan, and in part by Health and Labor Sciences Research Grants "Research on Regulatory Science of Pharmaceuticals and Medical Devices" from the Ministry of Health, Labour and Welfare, Japan. S.O. is a research resident of Japan Agency for Medical Research and Development. This work was also supported in part by the Takeda Science Foundation and by the Program for Leading Graduate Schools "HIGO" in Kumamoto University from MEXT. S.K. is a member of HIGO Program, MEXT, Japan.

\section{Authors' contributions}

SO conceived, designed experiments and acquired, analyzed, interpreted data and drafted manuscript. $\mathrm{HO}, \mathrm{MM}$, and NS acquired and analyzed data.
SK provided conceptual input, wrote the manuscript, and obtained funding. All authors read and approved the final manuscript.

\section{Competing interests}

The authors declared that they have no competing interests.

Ethics approval and consent to participate

Not applicable.

\section{Author details}

${ }^{1}$ School of Life Science and Technology, Tokyo Institute of Technology, 4259-B-25 Nagatsuta-cho, Midori-ku, Yokohama, Kanagawa 226-8501, Japan. ${ }^{2}$ Stem Cell Biology, Institute of Molecular Embryology and Genetics, Kumamoto University, Honjo 2-2-1, Kumamoto 860-0811, Japan. ${ }^{3}$ Division of Pharmacology, National Institute of Health Science, 1-18-1 Kamiyoga Setagaya-ku, Tokyo 158-8501, Japan.

Received: 19 January 2016 Accepted: 23 May 2016

Published online: 31 May 2016

\section{References}

1. Tam PP, Quinlan GA. Mapping vertebrate embryos. Curr Biol. 1996;6(2):104-6.

2. Ang SL, Wierda A, Wong D, Stevens KA, Cascio S, Rossant J, Zaret KS. The formation and maintenance of the definitive endoderm lineage in the mouse: involvement of HNF3/forkhead proteins. Development. 1993;119(4):1301-15.

3. Lawson KA, Pedersen RA. Cell fate, morphogenetic movement and population kinetics of embryonic endoderm at the time of germ layer formation in the mouse. Development. 1987;101(3):627-52.

4. Loh KM, Ang LT, Zhang J, Kumar V, Ang J, Auyeong JQ, Lee KL, Choo SH, Lim CY, Nichane $M$, et al. Efficient endoderm induction from human pluripotent stem cells by logically directing signals controlling lineage bifurcations. Cell Stem Cell. 2014;14(2):237-52

5. Nakanishi M, Kurisaki A, Hayashi Y, Warashina M, Ishiura S, Kusuda-Furue M, Asashima M. Directed induction of anterior and posterior primitive streak by Wnt from embryonic stem cells cultured in a chemically defined serum-free medium. FASEB J. 2009;23(1):114-22.

6. Lewis $\mathrm{SL}$, Tam PP. Definitive endoderm of the mouse embryo: formation, cell fates, and morphogenetic function. Dev Dyn. 2006;235(9):2315-29.

7. Alexander J, Stainier DY. A molecular pathway leading to endoderm formation in zebrafish. Curr Biol. 1999;9(20):1147-57.

8. Hudson C, Clements D, Friday RV, Stott D, Woodland HR. Xsox17alpha and -beta mediate endoderm formation in Xenopus. Cell. 1997;91(3):397-405.

9. Kanai-Azuma M, Kanai Y, Gad JM, Tajima Y, Taya C, Kurohmaru M, Sanai Y, Yonekawa H, Yazaki K, Tam PP, et al. Depletion of definitive gut endoderm in Sox17-null mutant mice. Development. 2002:129(10):2367-79.

10. Katsumoto K, Kume S. Endoderm and mesoderm reciprocal signaling mediated by CXCL12 and CXCR4 regulates the migration of angioblasts and establishes the pancreatic fate. Development. 2011;138(10):1947-55.

11. Nair S, Schilling TF. Chemokine signaling controls endodermal migration during zebrafish gastrulation. Science. 2008;322(5898):89-92.

12. Yasunaga M, Tada S, Torikai-Nishikawa S, Nakano Y, Okada M, Jakt LM, Nishikawa S, Chiba T, Era T, Nishikawa S. Induction and monitoring of definitive and visceral endoderm differentiation of mouse ES cells. Nat Biotechnol. 2005:23(12):1542-50.

13. Viotti M, Nowotschin S, Hadjantonakis AK. SOX17 links gut endoderm morphogenesis and germ layer segregation. Nat Cell Biol. 2014;16(12):1146-56.

14. Brown S, Teo A, Pauklin S, Hannan N, Cho CH, Lim B, Vardy L, Dunn NR, Trotter M, Pedersen R, et al. Activin/Nodal signaling controls divergent transcriptional networks in human embryonic stem cells and in endoderm progenitors. Stem Cells. 2011;29(8):1176-85.

15. McLean AB, D'Amour KA, Jones KL, Krishnamoorthy M, Kulik MJ, Reynolds DM, Sheppard AM, Liu H, Xu Y, Baetge EE, et al. Activin a efficiently specifies definitive endoderm from human embryonic stem cells only when phosphatidylinositol 3-kinase signaling is suppressed. Stem Cells. 2007:25(1):29-38.

16. Ogaki S, Morooka M, Otera K, Kume S. A cost-effective system for differentiation of intestinal epithelium from human induced pluripotent stem cells. Sci Rep. 2015;5:17297. 
17. Seguin CA, Draper JS, Nagy A, Rossant J. Establishment of endoderm progenitors by SOX transcription factor expression in human embryonic stem cells. Cell Stem Cell. 2008;3(2):182-95.

18. Mizoguchi T, Verkade H, Heath JK, Kuroiwa A, Kikuchi Y. Sdf1/Cxcr4 signaling controls the dorsal migration of endodermal cells during zebrafish gastrulation. Development. 2008;135(15):2521-9.

19. Stuckemann T, Wegleiter T, Stefan E, Nagele O, Tarbashevich K, Bock G, Raz E, Aanstad P. Zebrafish Cxcr4a determines the proliferative response to Hedgehog signalling. Development. 2012;139(15):2711-20.

20. Medof ME, Kinoshita T, Nussenzweig V. Inhibition of complement activation on the surface of cells after incorporation of decay-accelerating factor (DAF) into their membranes. J Exp Med. 1984;160(5):1558-78.

21. Lin F, Fukuoka Y, Spicer A, Ohta R, Okada N, Harris CL, Emancipator SN, Medof ME. Tissue distribution of products of the mouse decay-accelerating factor (DAF) genes. Exploitation of a Daf1 knock-out mouse and site-specific monoclonal antibodies. Immunology. 2001;104(2):215-25.

22. Ogaki S, Harada S, Shiraki N, Kume K, Kume S. An expression profile analysis of ES cell-derived definitive endodermal cells and Pdx1-expressing cells. BMC Dev Biol. 2011;11:13.

23. Shiraki N, Harada S, Ogaki S, Kume K, Kume S. Identification of DAF1/CD55, a novel definitive endoderm marker. Cell Struct Funct. 2010;35(2):73-80.

24. Shiraki N, Higuchi Y, Harada S, Umeda K, Isagawa T, Aburatani H, Kume K, Kume S. Differentiation and characterization of embryonic stem cells into three germ layers. Biochem Biophys Res Commun. 2009;381(4):694-9.

25. Shiraki N, Yoshida T, Araki K, Umezawa A, Higuchi Y, Goto H, Kume K, Kume S. Guided differentiation of embryonic stem cells into Pdx1-expressing regional-specific definitive endoderm. Stem Cells. 2008;26(4):874-85.

26. Bergmann A, Steller H. Apoptosis, stem cells, and tissue regeneration. Sci Signal. 2010;3(145):re8.

27. Berrier AL, Yamada KM. Cell-matrix adhesion. J Cell Physiol. 2007;213(3):565-73.

28. Diaferia GR, Jimenez-Caliani AJ, Ranjitkar P, Yang W, Hardiman G, Rhodes CJ, Crisa $\mathrm{L}$, Cirulli V. beta1 integrin is a crucial regulator of pancreatic beta-cell expansion. Development. 2013;140(16):3360-72.

29. Boettiger D. Mechanical control of integrin-mediated adhesion and signaling. Curr Opin Cell Biol. 2012;24(5):592-9.

30. Kim C, Ye F, Ginsberg MH. Regulation of integrin activation. Annu Rev Cell Dev Biol. 2011;27:321-45.

31. Ogaki S, Shiraki N, Kume K, Kume S. Wnt and Notch signals guide embryonic stem cell differentiation into the intestinal lineages. Stem Cells. 2013;31(6):1086-96.

32. Ruiz-Arguelles A, Llorente $\mathrm{L}$. The role of complement regulatory proteins (CD55 and CD59) in the pathogenesis of autoimmune hemocytopenias. Autoimmun Rev. 2007;6(3):155-61.

33. Lalli PN, Strainic MG, Lin F, Medof ME, Heeger PS. Decay accelerating factor can control T cell differentiation into IFN-gamma-producing effector cells via regulating local C5a-induced IL-12 production. J Immunol. 2007;179(9):5793-802.

34. Gadue P, Gouon-Evans V, Cheng X, Wandzioch E, Zaret KS, Grompe M, Streeter PR, Keller GM. Generation of monoclonal antibodies specific for cell surface molecules expressed on early mouse endoderm. Stem Cells. 2009;27(9):2103-13.

35. Tada S, Era T, Furusawa C, Sakurai H, Nishikawa S, Kinoshita M, Nakao K, Chiba T, Nishikawa S. Characterization of mesendoderm: a diverging point of the definitive endoderm and mesoderm in embryonic stem cell differentiation culture. Development. 2005;132(19):4363-74.

36. Katsumoto K, Fukuda K, Kimura W, Shimamura K, Yasugi S, Kume S. Origin of pancreatic precursors in the chick embryo and the mechanism of endoderm regionalization. Mech Dev. 2009;126(7):539-51.

37. Grossmann J. Molecular mechanisms of "detachment-induced apoptosisAnoikis". Apoptosis. 2002;7(3):247-60.

38. Higuchi Y, Shiraki N, Yamane K, Qin Z, Mochitate K, Araki K, Senokuchi T, Yamagata K, Hara M, Kume K, et al. Synthesized basement membranes direct the differentiation of mouse embryonic stem cells into pancreatic lineages. J Cell Sci. 2010;123(Pt 16):2733-42.

39. Brafman DA, Phung C, Kumar N, Willert K. Regulation of endodermal differentiation of human embryonic stem cells through integrin-ECM interactions. Cell Death Differ. 2013;20(3):369-81.

40. Chen AE, Borowiak M, Sherwood RI, Kweudjeu A, Melton DA. Functional evaluation of ES cell-derived endodermal populations reveals differences between Nodal and Activin A-guided differentiation. Development. 2013; 140(3):675-86
41. Tamplin OJ, Kinzel D, Cox BJ, Bell CE, Rossant J, Lickert H. Microarray analysis of Foxa2 mutant mouse embryos reveals novel gene expression and inductive roles for the gastrula organizer and its derivatives. BMC Genomics. 2008:9:511.

42. Benjamin JT, Gaston DC, Halloran BA, Schnapp LM, Zent R, Prince LS. The role of integrin alpha8beta1 in fetal lung morphogenesis and injury. Dev Biol. 2009;335(2):407-17.

43. Takada Y, Ye X, Simon S. The integrins. Genome Biol. 2007;8(5):215.

44. Turner DA, Rue P, Mackenzie JP, Davies E, Martinez Arias A. Brachyury cooperates with Wnt/beta-catenin signalling to elicit primitive-streak-like behaviour in differentiating mouse embryonic stem cells. BMC Biol. 2014;12:63.

45. Okita K, Ichisaka T, Yamanaka S. Generation of germline-competent induced pluripotent stem cells. Nature. 2007:448(7151):313-7.

46. Sakano D, Shiraki N, Kikawa K, Yamazoe T, Kataoka M, Umeda K, Araki K, Mao D, Matsumoto S, Nakagata N, et al. VMAT2 identified as a regulator of late-stage beta-cell differentiation. Nat Chem Biol. 2014;10(2):141-8.

\section{Submit your next manuscript to BioMed Central and we will help you at every step:}

- We accept pre-submission inquiries

- Our selector tool helps you to find the most relevant journal

- We provide round the clock customer support

- Convenient online submission

- Thorough peer review

- Inclusion in PubMed and all major indexing services

- Maximum visibility for your research

Submit your manuscript at www.biomedcentral.com/submit
Biomed Central 